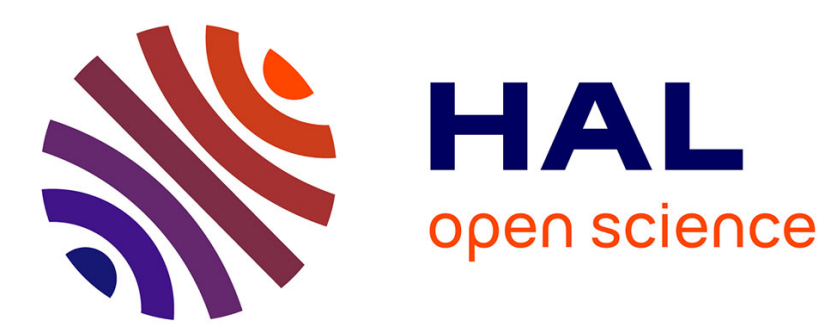

\title{
Velocity Dependent Adherence: An Analytical Approach for the JKR and DMT models
}

\author{
Etienne Barthel, Stéphane G. Roux
}

\section{To cite this version:}

Etienne Barthel, Stéphane G. Roux. Velocity Dependent Adherence: An Analytical Approach for the JKR and DMT models. Langmuir, 2000, 16, pp.8134. hal-00001370

\section{HAL Id: hal-00001370 \\ https://hal.science/hal-00001370}

Submitted on 29 Mar 2004

HAL is a multi-disciplinary open access archive for the deposit and dissemination of scientific research documents, whether they are published or not. The documents may come from teaching and research institutions in France or abroad, or from public or private research centers.
L'archive ouverte pluridisciplinaire HAL, est destinée au dépôt et à la diffusion de documents scientifiques de niveau recherche, publiés ou non, émanant des établissements d'enseignement et de recherche français ou étrangers, des laboratoires publics ou privés. 


\title{
Velocity Dependent Adherence: an Analytical Approach for the JKR and DMT models
}

\author{
Etienne Barthel and Stéphane Roux \\ Laboratoire CNRS/Saint-Gobain "Surface du Verre et Interfaces", \\ 39, quai Lucien Lefranc, BP 135, F-93303 Aubervilliers Cedex, France
}

(March 21, 2000)

\begin{abstract}
Recent Surface Forces Apparatus experiments on organic monolayers deposited on mica surfaces (Ruths, M. and Granick, S. Langmuir, 1998,14, 1804) have evidenced an increase of the pull-off force with driving velocity, which has been interpreted as due to local viscoelastic dissipation at the contact edge. Following Maugis and Barquins, this phenomenon is modelled through a combination of the Johnson-Kendall-Roberts equations for the adhesive contact of elastic bodies and the Gent-Schultz approach to viscoelastic crack dissipation. We provide an approximate analytical solution for the pull-off force as a function of the driving velocity which is supported by the result of direct numerical integrations of the equations in a large range of velocities. We discuss the application of this model to the adherence of two mica surfaces supporting surfactant monolayers.
\end{abstract}

Typeset using REVTEX 


\section{INTRODUCTION}

In recent papers, Ruths and Granick have studied the adhesive behaviour of various organic monolayers ${ }^{1,2}$ using a Surface Forces Apparatus (SFA). The monolayers were deposited on the SFA mica surfaces, and the pull-off force was measured as a function of the piezo driving velocity.

Although quantitatively different results were obtained depending upon the nature of the organic layer, the general result is that, typically, the pull-off force is constant for the lower velocity values. From this constant pull-off force, a so-called thermodynamic adhesion energy $w_{0}$ can be calculated. Then, for most of the monolayers studied, a mild (two- or threefold) increase in the pull-off force is observed when the driving velocity is increased (above 0.1 to $1 \mu \mathrm{ms}^{-1}$ ). In the case of a widely used surfactant, cetylamonium bromide (CTAB), it seems that this pull-off force increase, which is due to the onset of viscous dissipation at higher rates, can be accurately fitted to a power-law of the velocity.

Due to the lack of pull-off force increase in the case of glassy (i.e. solid-like) organic monolayer, it is clear that the dissipation is not due to the mica/glue/glass composite, but essentially originates from the process of separation of the two monolayers. Such an effect of viscous dissipation at the periphery of the contact zone on the adhesive contact of spheres has been investigated in the past ${ }^{3-7}$.

However, the results in these papers do not permit to calculate the pull-off force in the present experimental conditions easily. As a result, the above mentioned monolayer data $^{1}$ have been analyzed on a qualitative basis only. In fact, the authors assumed the driving velocity and the crack tip velocity (the speed at which the contact zone recedes) are proportional. It is clear, however, that the crack tip velocity increases markedly close to contact break-up, that is to say precisely when pull-off occurs and the pull-off force is measured.

The aim of the present paper is thus to provide a simple approximate analytical solution to the problem of pull-off in the presence of viscous dissipation at the crack tip and compare 
it with numerical results. We also show how this solution can be applied to analyse some data of Ref. ${ }^{1}$ concerning the adherence of mica surfaces supporting a surfactant monolayer.

\section{MODEL}

In the experiment, the lower sample of a rather soft (spring stiffness $k=1.110^{3} \mathrm{~N} / \mathrm{m}$ ) SFA is driven at a constant speed $V$ until the contact between the surfaces breaks up. The experiment is thus essentially force controlled, and the applied force is $P=k V t$ where $t$ is the time elapsed since the beginning of unloading. We aim at determining the variation of the contact radius $a$ with time. Following the standard approach, we use an elastic adhesive contact model (here $\mathrm{JKR}^{8}$ or $\mathrm{DMT}^{9}$ ) with a velocity-dependent adhesion. This approach is justified in the present case because the whole system behaves elastically, except the monolayers.

For the analytical calculations, the velocity dependent adhesion will be described by the Gent and Schultz equation ${ }^{10}$ which defines an effective velocity-dependent adhesion energy $w$ by

$$
w=w_{0}\left\{1+\left(\frac{1}{v_{0}} \frac{d a}{d t}\right)^{\beta}\right\}
$$

The main point is that this effective adhesion energy depends upon the crack front velocity through the characteristic velocity $v_{0}$ and the power law exponent $\beta$. Typical values for $\beta$ lie in the range 0.1 to $0.8^{7}$. To our knowledge, no explicit theory exists which justifies this form of adherence, although it has been argued that it stems from the distribution of characteristic relaxation times in the viscoelastic material ${ }^{6}$.

Of course, for the numerical approach, any other relevant form of dissipation may be used without any additional difficulty.

\section{A. Dimensionless formulation}

We introduce the compound normal surface rigidity 


$$
K^{-1}=\left\{\frac{E_{1}}{1-\nu_{1}^{2}}\right\}^{-1}+\left\{\frac{E_{2}}{1-\nu_{2}^{2}}\right\}^{-1},
$$

where the $E_{i}$ and $\nu_{i}$ stand for the Young modulus and Poisson ratio of solid $i$. We also define the compound curvature equal to $1 / R$ in the sphere-plane configuration, where $R$ is the sphere radius. Using Maugis' normalization ${ }^{11}$, the reduced contact radius reads:

$$
A \equiv a\left(\frac{\pi w_{0} R^{2}}{K}\right)^{-1 / 3},
$$

the reduced normal force

$$
\bar{P} \equiv \frac{P}{\pi w_{0} R}
$$

and the reduced penetration

$$
\Delta \equiv \delta\left(\frac{\pi^{2} w_{0}^{2} R}{K^{2}}\right)^{-1 / 3},
$$

In those notations, we can write the static solutions in the DMT and JKR limits as

$$
A^{3}= \begin{cases}\bar{P}_{0}+2 & (\mathrm{DMT}) \\ \bar{P}_{0}+\sqrt{6} A^{3 / 2} & (\mathrm{JKR}) .\end{cases}
$$

We also introduce the normalized driving velocity

$$
\alpha=\frac{k}{\left(\pi^{2} w_{0}^{2} R K\right)^{1 / 3}} \frac{V}{v_{0}}
$$

to express the differential equation to solve in both JKR and DMT limits

$$
\begin{aligned}
& A^{3}=\bar{P}+2\left(1+\left(\alpha \frac{d A}{d \bar{P}}\right)^{\beta}\right)(\text { DMT }) \\
& A^{3}=\bar{P}+\sqrt{6\left(1+\left(\alpha \frac{d A}{d \bar{P}}\right)^{\beta}\right)} A^{3 / 2}(\mathrm{JKR}) .
\end{aligned}
$$

Since the pull-off force in the absence of dissipation is

$$
\bar{P}_{p o, 0}= \begin{cases}2 & (\mathrm{DMT}) \\ 3 / 2 & (\mathrm{JKR}),\end{cases}
$$

the relative additional dissipative term is defined as

$$
\omega_{\text {diss }}=\frac{\bar{P}_{p o}-\bar{P}_{p o, 0}}{\bar{P}_{p o, 0}}
$$




\section{B. Numerical Solution}

Extracting the derivative, Eqs. 9 and 8 turn into first order differential equations which can be solved numerically by standard methods, like Runge-Kutta. Given the boundary condition (the initial contact radius), the contact radius at all time can be computed. In particular, in contrast to the equilibrium (static) case, the contact radius does go to zero at contact break-up for the simple form of dissipation given by Eq. 1. Indeed contact rupture at non zero contact radius would lead to infinite crack speed. But, in the present model, an increase in the crack speed increases the adhesion force, which leads to slowing down of the crack. It is precisely this balance that the present model describes.

Note that in this normalized form, the applied force is equivalent to time, and the pull-off force is thus simply the time it takes for the contact radius to go to zero.

In Fig. 1, we show the numerical results in the JKR case for the applied force (or time) as a function of the contact radius for a normalized driving velocity $\alpha$ of 0.05 and a dissipation exponent $\beta$ of 0.6 . The initial contact radius was taken as the equilibrium contact radius at zero load. The dissipationless JKR case is also shown for comparison. This figure examplifies the increase in pull-off force due to the viscous dissipation $\left(\omega_{\text {diss }}\right)$.

Typical dissipative contributions $\omega_{\text {diss }}$ computed numerically as a function of the driving velocity $\alpha$ for various intrinsic dissipation exponent $\beta$ are displayed on Fig. 2 for the JKR case. The general behaviour is clearly well approximated by a power law $\left(\omega_{\text {diss }}=\Omega \alpha^{\gamma}\right)$ at low velocity, although on this $\log -\log$ plot a definite curvature is observed at higher velocity for the larger values of $\beta$. The prefactor $\Omega$ and exponents $\gamma$ derived from a power law fit to these numerical data in the low velocity range are shown on Fig. 3 (markers) for the JKR case. In the DMT case, the power law behaviour gives an even more accurate description. The prefactor and exponents derived from the fit are shown on Fig. 4. Generally speaking, the prefactor is only slightly dependent upon the dissipation exponent, while the pull-off force exponent is close to $\beta$ for values smaller than about 0.5 and becomes increasingly larger beyond this limit. 


\section{Approximate Analytical Solution}

The quasi-static solution, which consists in using the static solution $\bar{P}_{0}(A)$ (Eq. 6) to compute $d A / d \bar{P}$ in Eq. 9 provides a good approximation for the early stage of the unloading if the pulling velocity is not too large (Fig. 1). This explains why the pull-off force is independent of the initial contact radius in a wide range of velocities and dissipation exponents. However, as the static contact break-up force is approached, the static solution gives a diverging $d A / d \bar{P}$, and hence however small the pulling velocity, the quasistatic solution breaks down.

Indeed, inspection of Eq. 9 shows that the order of the differential equation changes when $\alpha$ goes to zero: the perturbation is singular. This is the reason why the $\alpha=0$ (static) curve is not a good approximation to the $\alpha \neq 0$ solutions for an arbitrarily small $\alpha$.

Therefore, we focus on the region close to contact break-up (where the crack speed is large), and search for an expansion in integer powers of the contact radius variation $A-A_{0}$ of the static solution $\bar{P}_{0}(A)$ for small driving velocity $\alpha$ close to the point of static contact breakup $A_{0}$. Due to the singularity in the problem, we allow for singular coefficients (behaving as power law with $\alpha$ ) in the expansion.

In the DMT case, contact break-up occurs at zero contact radius. We will thus simply expand Eq. 8 for small $A$ starting from the form

$$
\bar{P}=A^{3}-2\left(1+\left(\frac{1}{\alpha} \frac{d \bar{P}}{d A}\right)^{-\beta}\right) .
$$

In the JKR case, contact break-up (for a soft measuring system) occurs at

$$
A_{0}=\left(\frac{3}{2}\right)^{1 / 3}
$$

We expand around this point starting from a second order approximation to the static solution. With the notation

$$
\delta A=A-A_{0}
$$

we obtain the approximate static solution 


$$
\bar{P}_{0}=-\frac{3}{2}+\left(\frac{3}{2} \delta A\right)^{2}
$$

and the approximate differential equation

$$
\bar{P}=\left(\frac{3}{2} \delta A\right)^{2}-\frac{3}{2}\left(1+\left(\frac{1}{\alpha} \frac{d \bar{P}}{d \delta A}\right)^{-\beta}\right) .
$$

Comparison of Eq. 12 and 16 suggests that the DMT and JKR cases are very similar, the only difference occuring in the exponent in the first term of the right-hand side member.

Let us now tackle the JKR case. We consider the expansion

$$
\bar{P}=-\frac{3}{2}+\sum_{i \geq 0} c_{i} \alpha^{\gamma_{i}} \delta A^{i} .
$$

Inserting this expression in Eq. 16 and expanding the right-hand side up to the order 2 in $\delta A$, yields through identification:

$$
\left\{\begin{array}{l}
\gamma_{0}=2 \beta /(2+\beta) \\
\gamma_{1}=\beta /(2+\beta) \\
\gamma_{2}=0
\end{array}\right.
$$

and

$$
\left\{\begin{array}{l}
c_{0}=-\frac{3}{2}\left(\frac{3}{2} \beta c_{2}\right)^{-\beta /(2+\beta)} \\
c_{1}=\left(\frac{3}{2} \beta c_{2}\right)^{1 /(2+\beta)} \\
c_{2}=\frac{9}{2(3+\beta)}
\end{array}\right.
$$

For small $\delta A$, the main additional contribution to the force is thus the zeroth order term. As evidenced by the numerical calculations (Fig. 1), the last stage in the contact rupture (when the contact radius is significantly smaller then $A_{0}$ ) is fast, and thus - due to the force-time equivalence - does not lead to a major additional increase in the pull-off force. Thus, the dissipative contribution to the effective adhesion energy can be approximately written, in the JKR case:

$$
\omega_{\text {diss. }}=\left[\frac{27 \beta}{4(3+\beta)}\right]^{-\frac{\beta}{2+\beta}} \alpha^{\frac{2 \beta}{2+\beta}} \quad(\mathrm{JKR})
$$


The DMT case can be treated in a similar way. Due to the presence of an exponent 3 in Eq. 12 instead of 2 in Eq. 16, it requires an expansion of the right-hand side up to the order 3. Similarly, we obtain

$$
\omega_{\text {diss. }}=\left[\frac{(\beta+2)(2 \beta+3)}{24 \beta^{2}}\right]^{\frac{\beta}{3+2 \beta}} \alpha^{\frac{3 \beta}{3+2 \beta}} \quad(\mathrm{DMT}) .
$$

\section{Comparison of analytical and numerical results}

The approximate results for the radius of contact vs. force (or time) are compared to the numerical results in Fig. 1. As expected, the approximate curves do reflect an increase in the pull-off force similar to the increase observed numerically, but of course do not suitably model the contact behaviour away from the expansion point.

The agreement for the pull-off force can be quantified by plotting a comparison between the analytical and the numerical results for the prefactor $\Omega$ and the exponent $\gamma$ of the power law increase of the dissipative contribution to the effective adhesion energy with driving velocity (Fig. 3 for the JKR case; Fig. 4 for the DMT case). The agreement is especially good for the exponent in both cases. For lower values the intrinsic $(\beta)$ and apparent dissipation exponents are similar. Non negligible deviations appear above $\beta \simeq 0.3$, with the apparent exponent being somewhat smaller than the intrinsic one. As for the prefactor, which is about 1 and does not show large variations, the agreement is good in the DMT case, but qualitative only in the JKR case for larger values of $\beta$ (Fig. 3, top). This prefactor is the major discrepancy observed between the numerical solutions and the analytical approach at values of $\beta$ larger than 0.5 (Fig. 2), and a constant prefactor $\Omega=1.1$ seems to provide a better (and simpler) approximation in the intermediate $\beta$ range in the JKR case.

Thus, within these limitations, the approximate model can be used to assess pull-off forces which turn out to depend upon the driving velocity as a power law. In addition, naturally, to the thermodynamic contribution to the adhesion energy $w_{0}$, two parameters can thus be obtained from the data: the intrinsic dissipation exponent $\beta$, and the characteristic crack 
speed velocity $v_{0}$ which characterize the dissipative process at the crack tip according to Eq. 1.

Let us note that a similar treatment can be simply extended to the case of any surface interactions ${ }^{11,12}$ interpolating between JKR (limit of vanishing range of interaction) and DMT (limit of infinite interaction range). In such a case, the static pull-off force displays a maximum at a non-zero contact radius, and the static solution can be approximated by a parabola close to this point. Therefore a similar result as the one obtained for JKR should hold, i.e. same power-law for the pull-off force as a function of drive velocity, but with modified prefactors.

In one of his last contributions, V. Muller has tackled a closely related problem ${ }^{7}$. However, he considered the case where the displacement velocity $\dot{\delta}$ is constant, as happens for an infinitely rigid measuring system working at constant driving velocity. His approach essentially relies on neglecting the thermodynamic term in Eq. 1, using a JKR model and approximate relation

$$
\frac{d \delta}{d a} \propto \frac{a}{R}
$$

which can be justified on geometrical grounds, and is probably only valid for moderate adherence. The resulting scaling exponent for the force is $3 \beta /(3+\beta)$, which is naturally rather different from the exponent we obtained for the case of a force controled ( $\dot{F}$ constant) set-up, with consideration for the thermodynamic adhesive contribution.

\section{APPLICATION TO CTAB MONOLAYERS}

\section{A. Dissipative Contribution}

We now reinvestigate the $\mathrm{CTAB}$ monolayer experiments by Ruths \& Granick ${ }^{1}$. Note that among the various types of monolayers probed in these experiments, CTAB turned out to be the only system for which a power law dissipative process seemed to apply. For a different form of dissipation, a suitable substitute for Eq. 1 should be provided. The CTAB 
adherence data were gathered letting the system sit for different contact times prior to the pull-off force measurements ${ }^{1}$. The data for the effective adhesion energy, or adherence, computed from the experimental results through a JKR expression for various contact times are displayed on Fig. 5. These data clearly exhibit the singular behaviour with the driving velocity discussed previously.

Following the authors in describing the type of contact as JKR, we use our power law expression Eq. 20 with a constant prefactor equal to 1.1, combined with Eqs. 4 and 11 to fit the data (Fig. 5), or

$$
\omega=\omega_{0}\left(1+1.1 \alpha^{2 \beta /(2+\beta)}\right)
$$

with $\alpha$ defined by Eq. 7. During the fit, we excluded the points exhibiting an adherence larger than twice the static value, since our analytical expression is less accurate at larger driving velocity. On the other hand, we have included the static adhesion energy as a free fitting parameter, so that the total number of fitting parameters is 3 . Although it is clearly possible, we did not attempt fits to the full numerical solution to Eq. 9, essentially because the experimental points are rather scattered for large values of the driving velocity.

The resulting thermodynamic adhesion energy, intrinsic dissipation exponent and characteristic velocity $v_{0}$ as a function of contact time are displayed in the Table.

In their paper, Ruths \& Granick give a rough estimate (0.5) of the apparent pull-off exponent (characteristic of the power law dependence of the velocity-dependent part of the pull-off force upon the driving velocity). Our fit to the data gives a value closer to 0.45 for the same parameter.

The present approach, however, allows for a more quantitative treatment: we typically find a value of $0.60-0.65$ for the intrinsic dissipation exponent except at the smallest contact time of $10 \mathrm{~ms}$, where the intrinsic exponent seems definitely lower (0.5).

This case illustrates the way the physical exponent $\beta$ may be somewhat underestimated if the crack tip velocity and the driving velocity are assumed equivalent.

We now turn to the characteristic velocity (Fig. 6). It is in the range of $\mathrm{mm} / \mathrm{s}$, and 
exhibits a variation with contact time to the power -0.3 roughly.

Within experimental noise, the present analysis seems to identify two phenomena: 1) for very short contact times, the intrinsic dissipation exponent is lower; 2) the characteristic velocity for the onset of dissipation decreases with contact time. These phenomena probably result from the shift to different dissipation modes with time due to interdigitation of the CTAB chains grafted on both surfaces.

\section{B. Limitations}

Some limitations in the description of the Ruths \& Granick data should however be mentioned.

First, for most of the chemicals investigated, the pull-off force increase is not a power law. This clearly invalidates the application of the Gent-Schultz description (Eq. 1). Within the experimental noise, one should figure out the relevant form of dissipation.

Second, it is specified in ${ }^{1}$ that in the case of CTAB, pull-off occurs at non-zero contact radius, which is correct in the JKR model, but not consistent with the present approach (cf. II B). This is probably due to the fact that the power law dissipation is valid only over a limited range of crack velocity. If the effective adhesion energy, above a given velocity, decreases again, then the contact is again unstable and the pull-off will occur once this crack velocity has been reached ${ }^{13}$.

Third, it is very surprising to note that, for some chemicals (polybutadiene) the experimental system seemed to be stable for minutes with contact radii significantly smaller than the nominal JKR equilibrium contact radius (at fixed load). This would only be expected if the range of interaction of surface forces extends over a non-zero distance (as assumed e.g. in the DMT approach). This may suggest that a generalization of the adhesive contact mechanics to intermediate range interaction ${ }^{11,12}$ may be appropriate. 


\section{CONCLUSION}

Under the assumption of a Gent-Schultz type of crack tip dissipation, we have given an approximate expression for the dissipative contribution to the pull-off force which compares favorably with numerical calculations. The intrinsic dissipation exponent is shown to be rather sensitive to the data treatment and the accuracy of the model applied, except for low values (smaller than 0.5 , roughly). In addition, valuable information can be extracted from the characteristic velocity above which dissipative phenomena start to play a role. Reinvestigating the CTAB data by Ruths and Granick $^{1}$, we have shown that the actual intrinsic dissipation exponent is only slightly larger than the estimate they proposed, while the characteristic velocity decreases with contact time $t$ roughly as $t^{-0.3}$.

\section{ACKNOWLEDGMENTS}

We thank M. Ruths and S. Granick for communication of the data and discussion. 


\section{REFERENCES}

(1) Ruths, M. and Granick, S. Langmuir, 1998, 14, 1804.

(2) Ruths, M. and Granick, S. J. Phys. Chem. B, 1998, 102, 6056.

(3) Maugis, D. and Barquins, M. J. Phys. D.: Appl. Phys., 1978, 11, 1989.

(4) Fuller, K. N. and Roberts, A. D. J. Phys. D:Appl. Phys., 1981, 14, 221.

(5) Greenwood, J. A. and Johnson, K. L. Phil. Mag., 1981, 43, 697.

(6) Maugis, D. J. Adhesion Sci. Tec., 1987, 1, 105.

(7) Muller, V. M. Colloid Journal, 1996, 58, 612.

(8) Johnson, K. L., Kendall, K., and Roberts, A. D. Proc. Roy. Soc. London A, 1971, 324, 301.

(9) Derjaguin, B. V., Muller, V. M., and Toporov, Yu. P. J. Colloid Interface Sci., 1975, $53,314$.

(10) Gent, A. and Schultz, J. J. Adhesion, 1972, 3, 281.

(11) Maugis, D. J. Colloid Interface Sci, 1992, 150, 243.

(12) Barthel, E. J. Colloid Interface Sci., 1998, 200, 7.

(13) Maugis, D. In Adhesion; Allen, K. W. Ed; Elsevier, 1988. 


\begin{tabular}{|c|c|c|c|c|}
\hline contact time t $(\mathrm{s})$ & 0.01 & 1.0 & 100 & 500 \\
\hline adhesion energy $w_{0}\left(\mathrm{mJm}^{-2}\right)$ & 48.7 & 48.7 & 45.0 & 44.4 \\
\hline intrinsic exponent $\beta$ & 0.47 & 0.65 & 0.59 & 0.65 \\
\hline characteristic velocity $v_{0}\left(\mathrm{mms}^{-1}\right)$ & 10.5 & 2.2 & 0.7 & 0.3 \\
\hline
\end{tabular}

Table: Thermodynamic adhesion energy, intrinsic dissipation exponent and characteristic velocity for the onset of dissipation derived from the pull-off force data on CTAB monolayers ${ }^{1}$ through the approximate model Eq. 23. 


\section{Captions:}

Fig. 1: Normalized normal force vs. normalized contact radius for a normalized driving velocity $\alpha=.05$ and an intrinsic dissipation exponent $\beta=0.6$ as obtained from the numerical integration (thick line). The JKR case ( $\alpha=0$; thin line) is also shown to illustrate the increase of the pull-off force for non zero values of $\alpha$. The thin dashed line is the quasistatic approximation while the thick dashed curve is the analytical approximation obtained through singular expansion around the JKR pull-off point (see text).

Fig. 2: Dissipative contribution to the adhesion energy vs. normalized driving velocity $\alpha$ (markers) as a function of the intrinsic dissipation exponent $\beta$ (from the numerical integration) for a JKR model including crack tip dissipation. The solid lines are the predictions from the model.

Fig. 3: Prefactor and effective (pull-off force) exponent as a function of the intrinsic dissipation exponent $\beta$ for a JKR model including crack tip dissipation. Results obtained from the power law fits to the numerical results (cf. Fig. 2)(symbols) and from the singular expansion model (solid lines) are compared.

Fig. 4: Prefactor and effective (pull-off force) exponent as a function of the intrinsic dissipation exponent $\beta$ for a DMT model including crack tip dissipation. Results obtained from the power law fits to the numerical results (symbols) and from the singular expansion model (solid lines) are compared.

Fig. 5: Fits (solid lines) to the CTAB data (symbols) from Ruths \& Granick ${ }^{1}$ using the approximate analytical solution for a JKR model including crack tip dissipation (as a function of contact time: $0.01,1.0,100.0$ and 500.0 s.). The curves are offset along the velocity axis for clarity. Due to the limited range of validity of the approximation, the fits rely on the low effective adhesion energy part of the data only. They make it possible to determine the thermodynamic adhesion energy $\omega_{0}$, the characteristic dissipation velocity $v_{0}$ and the intrinsic dissipation exponent $\beta$ (Table).

Fig. 6: Characteristic velocity $v_{0}$ of viscous dissipation onset vs. contact time, as obtained from the fits (cf. Fig. 5). The characteristic velocity is in the $\mathrm{mm} / \mathrm{s}$ range and 
within the experimental uncertainty, decreases with contact time to the power -0.3 . 


\section{FIGURES}

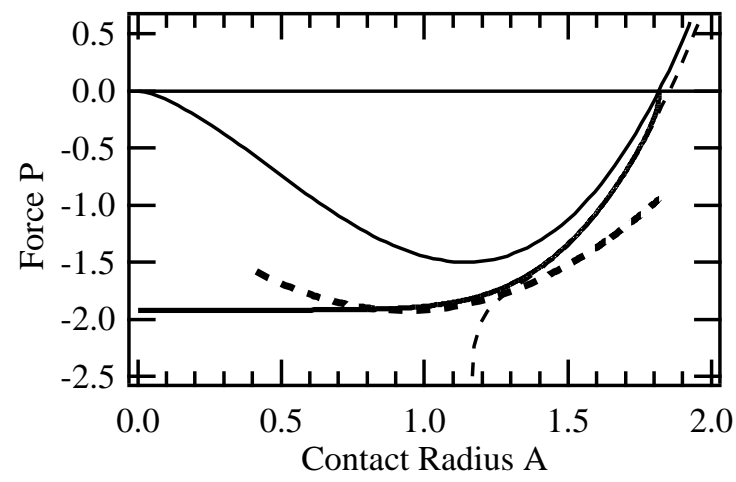

FIG. 1. Barthel \& Roux 


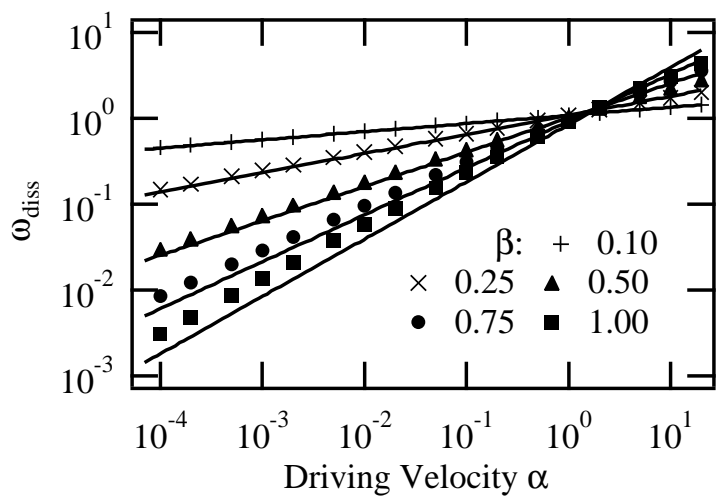

FIG. 2. Barthel \& Roux 


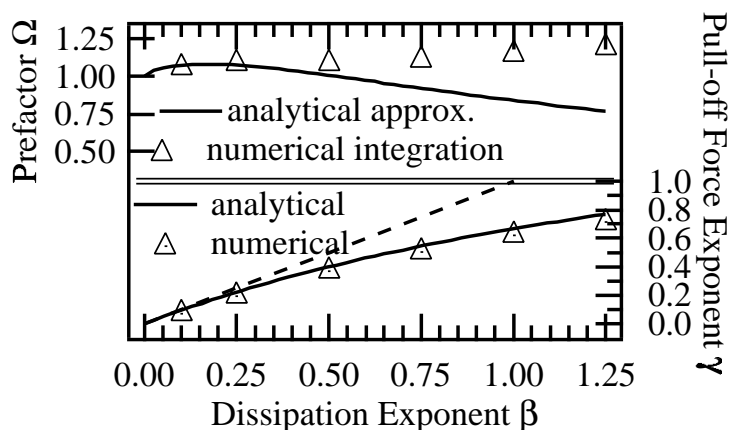

FIG. 3. Barthel \& Roux 


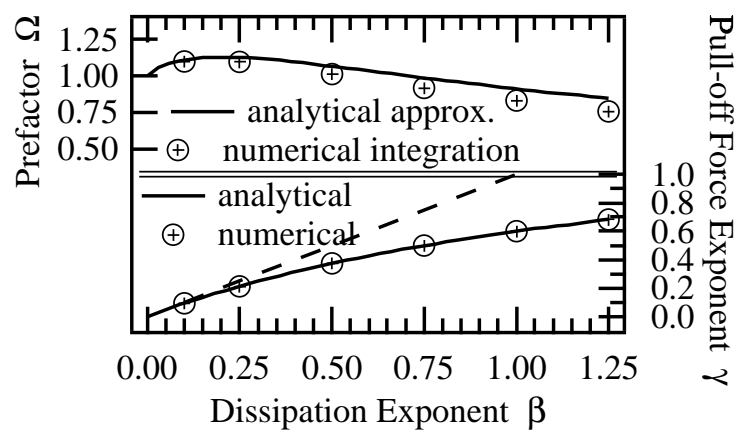

FIG. 4. Barthel \& Roux 


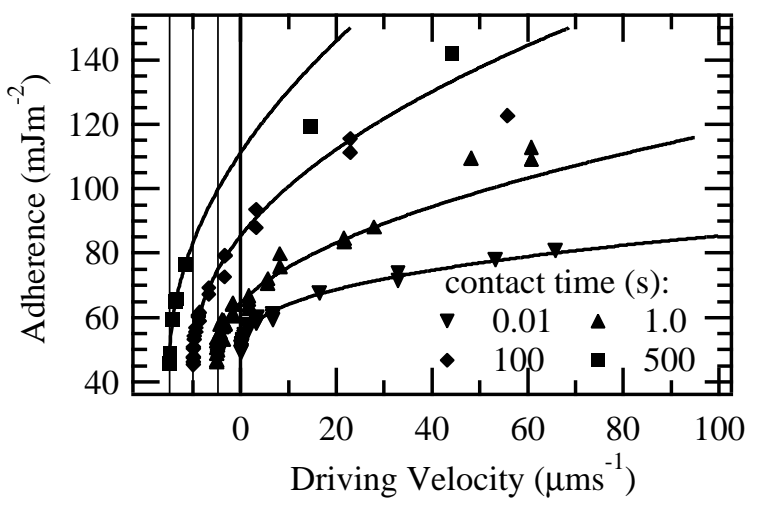

FIG. 5. Barthel \& Roux 


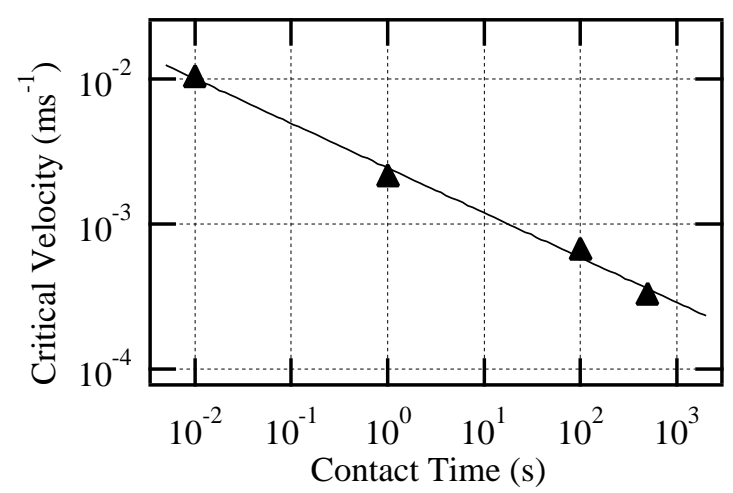

FIG. 6. Barthel \& Roux 